\title{
Golden Proportion in Different Types of Smile: A Photographic Evaluation
}

\author{
Shubhangi Jain ${ }^{1 *}$, Payal Sharma ${ }^{2}$, Divya Shetty ${ }^{3}$ and Kumar Adarsh ${ }^{4}$ \\ ${ }^{1}$ Department of Orthodontics and Dentofacial Orthopaedics, ITS Dental College, Muradnagar, Ghaziabad India \\ ${ }^{2}$ Department of Orthodontics and Dentofacial Orthopaedics, ITS Dental College, Muradnagar, Ghaziabad, India \\ ${ }^{3}$ Department of Orthodontics and Dentofacial Orthopaedics, ITS Dental College, Muradnagar, Ghaziabad, India \\ ${ }^{4}$ Dr BR Ambedkar Dental College, Patna, India \\ *Corresponding Author: Shubhangi Jain, Department of Orthodontics and Dentofacial Orthopaedics, ITS Dental College, Muradnagar, \\ Ghaziabad India.
}

Received: October 22, 2019; Published: November 18, 2019

DOI: $10.31080 /$ ASDS.2019.03.0703

\begin{abstract}
Introduction: The purpose of the study was to evaluate variation of mathematical proportions between widths of maxillary anterior teeth from the golden proportion in patients with narrow, average and wide smile.

Methods: Standardised frontal smiling photographs of 30 patients with aesthetically pleasing smile were taken. The buccal corridor widths and inter-commissure distance were measured. The patients were divided into 3 groups according to buccal corridor width, Wide (buccal corridor $<13 \%$ ), narrow (buccal corridor $>17 \%$ ) and average (buccal corridor $13-17 \%$ ). Widths of the maxillary anterior teeth were measured using a software scope version 4.0 (USA). The mathematical proportions between central and lateral incisors and lateral incisors and canine were calculated. The obtained proportions were compared with the golden proportions.

Results: A significant variation from the golden proportion was found in all smile group in proportions of both the lateral incisor and canine. This variation was maximum in wide smile for both lateral incisor as well as canine followed by narrow smile. The least deviation from golden proportion was seen in average smile patient.

Keywords: Smile; Esthetics; Orthodontics; Golden Proportion
\end{abstract}

\section{Abbreviations \\ GP: Golden Proportion}

\section{Introduction}

The measurement of human face since the greek era has aroused interest of anatomists, scientists, dentists and even artists. The modern anthropometry identifies the realistic sizes and proportions as opposed to the classic times [1].

In the modern society, people seek treatment with primary aim to improve their smile esthetics. There are several reference parameters that are to be followed to achieve an esthetic smile [2].

Therefore to alter dentofacial form, whether through facial growth modification, orthodontics or surgery, a knowledge and understanding of these parameters of facial beauty, facial esthetics, proportions and symmetry [3].

Creating harmonious proportions between the widths of maxillary anterior teeth when restoring or replacing them is one of the major tasks in esthetic dentistry [4].
The golden ratio arises from dividing a line segment so that the ratio of the whole segment to the large piece is equal to the ratio of the larger piece to the smaller piece. So if the bigger segment is $X$ then $X=.618033 \ldots$ and $1 / X=1.618033$. Commonly the letter $\Phi$ is used to represent the golden ratio 1.61803 [5].

The golden proportion was used by ancient Egyptians and described geometrically by Euclid. This proportion is associated with aesthetics and harmony in several fields such as architecture, sculpture, music, poetry and human face. Lombardi first noted that the teeth had a harmonious proportion to the adjacent teeth, however he also stated that the golden proportion was too strong for determining tooth size [6].

Levin suggested the use of the theory of Golden proportion to relate the successive width of the anterior teeth, as viewed from the labial aspect. He said that the "width of the central incisor should be in golden proportion to the width of the lateral incisor and that the lateral incisor should be in golden proportion to the width of the canine, when viewed from the front". [7]. 
Snow stated that symmetry, dominance, and proportion for an esthetically pleasing smile are three composition elements required to create unity and esthetics in a smile and the concept of the golden percentage is a useful application in the diagnosis and development. He proposed a golden percentage in which the proportional width of each tooth should follow from right to left canine $10 \%$, lateral $15 \%$, central $25 \%$, central $25 \%$, lateral $15 \%$, and canine $10 \%$ of the total distance across the anterior segment, to get an esthetically pleasing smile.

He considered golden percentage to be very useful as it does not depend on lateral incisors alone for analysis, asymmetry is easily identifiable and can be easily analyzed chairside. He also stated that the curvature of the arch form was a very important factor in esthetics of the tooth proportions. He observed that the narrower arches followed golden proportion greater than wider arches. Some other authors have also mentioned the use of this proportion in anterior esthetics $[7,8]$. On the other hand few studies including a few done on south Asian population have shown that the golden proportion was rarely seen in maxillary anteriors [8-11].

Other disciplines in dentistry have incorporated many concepts in smile aesthetics including the presence of buccal corridor space. It is the negative space created between the buccal surfaces of the posterior teeth and the inner wall of the cheek when the patient smiles [12].

The size of buccal corridor and arch width determine the width of the smile. According to Yang., et al. The buccal corridor itself is affected by factors such as growth pattern, incisor exposure, inter premolar width and tooth material [13].

According to Profit, the buccal corridor width in ideal smile is $13-17 \%$ of the intercommisure distance, less than $13 \%$ in wide smiles and greater than $17 \%$ in narrow smiles [14].

The arch width and arch form can be modified by the orthodontic treatment.

Few studies established a relation between esthetic proportions and arch width and a few have established relation between tooth material and inter premolar width and buccal corridor width.

Till now no studies have been conducted to assess the correlation between golden proportion and the smile width. Therefore, the purpose of this study was to evaluate the proportion of the maxillary anterior teeth in patients with narrow, average and wide smiles and their variation from the golden proportion.

\section{Material and Method}

The present study was conducted in ITS-CDSR, Murad agar, UP, India in patients with aesthetically pleasing smile. Ethical approval was obtained from the ITS-CDSR ethics committee. 60 subjects were selected on the following inclusion criteria.

\section{Inclusion criteria}

- Subjects of 15 - 25 years of age.

- Patients were to have all their natural teeth.

- No History of dental anomalies associated with tooth size and morphology alteration.

- No history of orthodontic treatment.

\section{Exclusion criteria}

- Teeth having maxillary anterior restorations or history of trauma or maxillofacial surgery.

- Presence of moderate to severe rotation, spacing or crowding.

- Presence of severe dentofacial deformities or obvious asymmetries.

Criteria for Standardization of Photographs

- All photographs were taken with a natural head posture and with the interpupillary line parallel to the floor.

- The camera was positioned on a stand at the same distance for each photograph, so that the line of the central lens to the eye was parallel to the horizontal plane and the lens was centered between both eyes.

- Two electronic flashes on the stands were placed at an angle of 45 degrees slightly above the patient, to ensure equal lighting on both sides of the face, to avoid the shadow or darkness on face.

- A clear white background was used for taking photographs in the study.

- The facial photographs were evaluated by a panel of judges comprising of 5 orthodontists and 30 photographs were selected based on the inclusion criteria.

All the photographs were cropped using Picasa 3.9.137. 163 (USA) to a standard size and $4 \times 6$ ratio. The measurements on the photographs were done using scope version 4.0 (USA).

The buccal corridors (Figure 2 and 3 ) and the inter-commissure distance (Figure 1) was measured on the photographs and the sample was divided into three groups as follows: 
- $\quad$ Buccal corridor width $13-17 \%$ - average smile (Figure 4)Group I.

- Buccal corridor width $<13 \%$ - wide smile (Figure 5)- Group II.

- Buccal corridor width $>17 \%$ - narrow smile (Figure 6) - Group III.

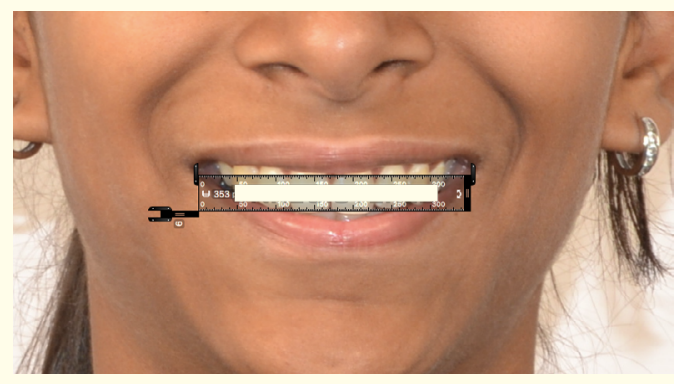

Figure 1: Measuring the inter-commissure width.

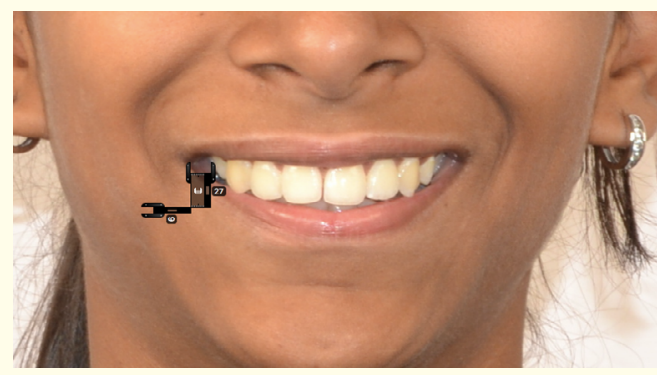

Figure 2: Measuring the right buccal corridor.

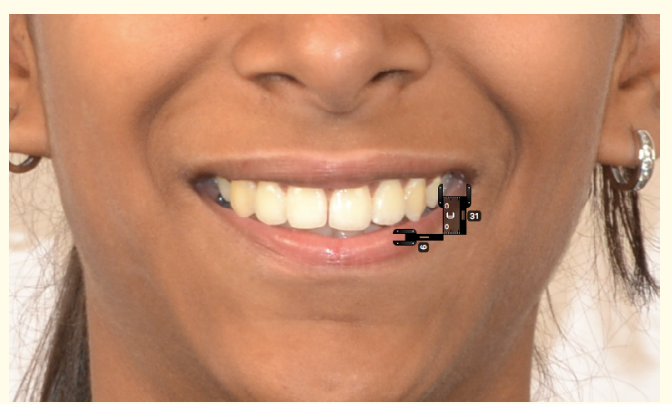

Figure 3: Measuring the left buccal corridor width.

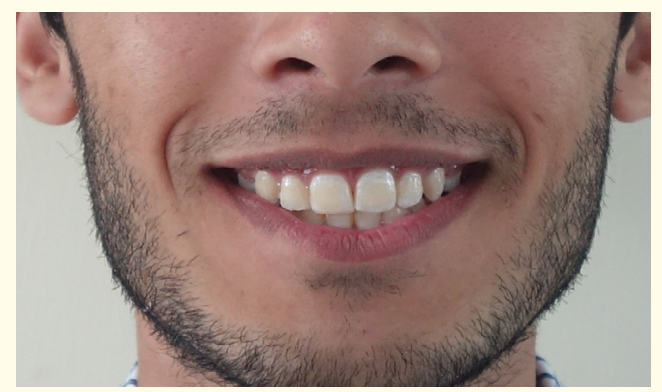

Figure 4: Average smile

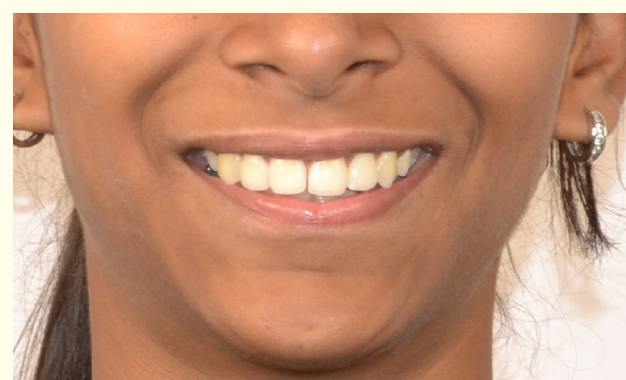

Figure 5: Wide smile.

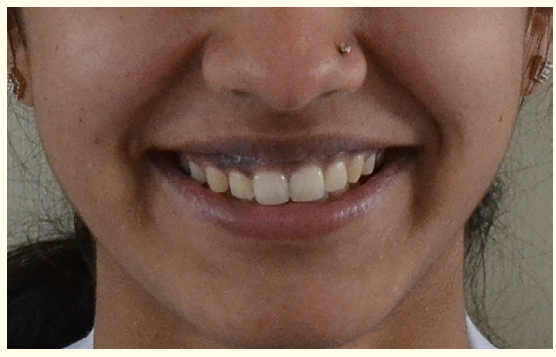

Figure 6: Narrow smile.

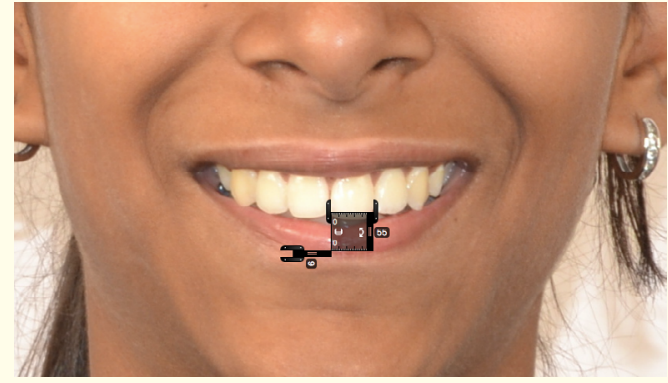

Figure 7: Measuring the central Incisor width.

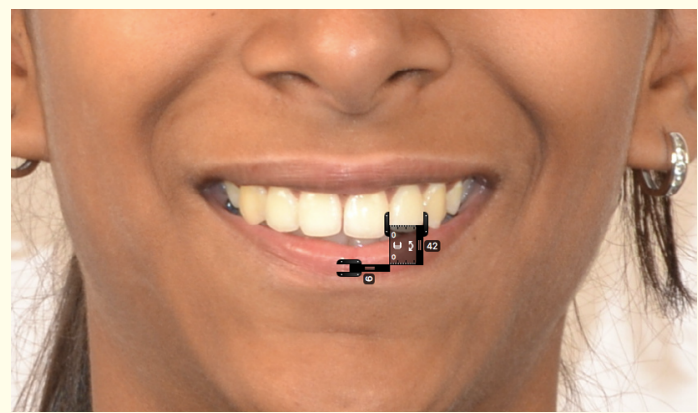

Figure 8: Measuring the lateral incisor width. 


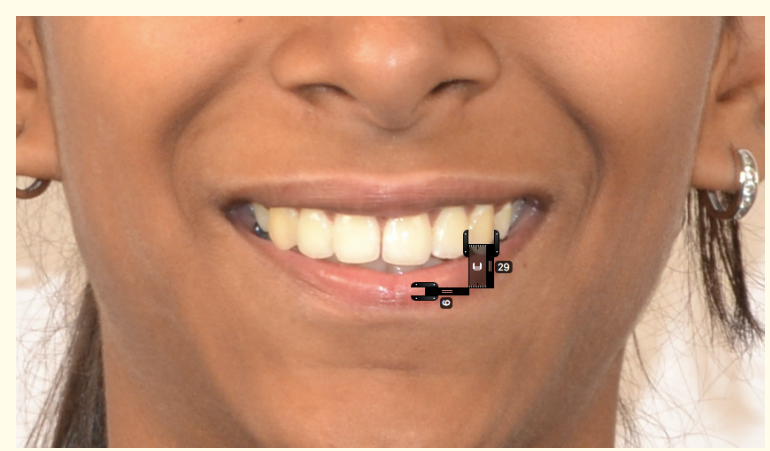

Figure 9: Measuring the canine width.

The widths of the maxillary central incisor, lateral incisor and canine were also measured.

The proportions of the lateral to central incisor and lateral incisor to canine were calculated for all 30 patients.

The results were tabulated and subjected to statistical analysis (The SPSS 20.0, SPSS Inc, Chicago, Illinois)
The descriptive statistics, including the means, standard deviations, minimum and maximum values were calculated for each group.

Degree of linear relationship between the variables in each group was determined by using the one way ANOVA. $\mathrm{P}<0.05$ was considered to be a statistically significant difference.

\section{Results and Discussion Results}

The oneway ANOVA test revealed a statistically significant difference of the proportions of maxillary anterior teeth from the golden proportion in all three groups.

A post hoc Bonferroni test was done that revealed that a statistically significant difference was found in all groups except lateral incisor and canine proportion in average smile groups.

The greatest difference from the golden proportion was found in wide smiles followed by narrow smile and average smile.

\begin{tabular}{|l|c|c|c|c|}
\hline & N & Mean & Std. Deviation & Std. Error Mean \\
\hline Canine Proportion Difference from GP In Average Smile Group & 10 & $5.6200 \%$ & $2.24935 \%$ & $0.71131 \%$ \\
\hline Lateral Incisor Proportion Difference from The GP In Average Smile Group & 10 & $4.9230 \%$ & $3.66433 \%$ & $1.15876 \%$ \\
\hline Lateral Incisor Proportion Difference from The GP In Wide Smile Group & 10 & $9.1100 \%$ & $6.17314 \%$ & $1.95212 \%$ \\
\hline Canine Proportion Difference from GP In Wide Smile Group & 10 & $10.2700 \%$ & $5.25231 \%$ & $1.66093 \%$ \\
\hline Lateral Incisor Proportion Difference from The GP In Narrow Smile Group & 10 & $7.8900 \%$ & $4.70849 \%$ & $1.48896 \%$ \\
\hline Canine Proportion Difference from GP In Narrow Smile Group & 10 & $9.4600 \%$ & $2.91860 \%$ & $0.92294 \%$ \\
\hline
\end{tabular}

Table 1: GP= Golden proportion.

\begin{tabular}{|l|c|c|c|c|c|}
\hline & Sum of Squares & Df & Mean Square & F & Sig. \\
\hline Between Groups & 767.919 & 6 & 127.987 & 7.801 & .000 \\
\hline Within Groups & 1033.555 & 63 & 16.406 & & \\
\hline Total & 1801.474 & 69 & & & \\
\hline
\end{tabular}

Table 2: ANOVA test to evaluate the significance of intergroup and intragroup difference.

\begin{tabular}{|c|c|c|c|c|c|c|}
\hline \multicolumn{7}{|c|}{ Multiple Comparisons } \\
\hline \multicolumn{7}{|c|}{ Dependent Variable: GP Bonferroni } \\
\hline \multirow{2}{*}{ (I) S No } & \multirow{2}{*}{ (J) $S$ No } & \multirow{2}{*}{$\begin{array}{c}\text { Mean } \\
\text { Difference (I-J) }\end{array}$} & \multirow{2}{*}{ Std. Error } & \multirow{2}{*}{ Sig. } & \multicolumn{2}{|c|}{ 95\% Confidence Interval } \\
\hline & & & & & Lower Bound & Upper Bound \\
\hline \multirow{6}{*}{$\begin{array}{l}\text { Golden } \\
\text { proportion }\end{array}$} & Lateral incisor proportion in narrow smile group & $-7.8900000^{*}$ & 1.8113881 & .001 & -13.625100 & -2.154900 \\
\hline & Lateral incisor proportion in wide smile group & $-9.1100000^{*}$ & 1.8113881 & .000 & -14.845100 & -3.374900 \\
\hline & Lateral incisor proportion in average smile group & -4.9200000 & 1.8113881 & .179 & -10.655100 & .815100 \\
\hline & Canine proportion in narrow smile group & $-9.4600000^{*}$ & 1.8113881 & .000 & -15.195100 & -3.724900 \\
\hline & Canine proportion in wide smile group & $-10.2700000^{*}$ & 1.8113881 & .000 & -16.005100 & -4.534900 \\
\hline & Canine proportion in average smile group & -5.6200000 & 1.8113881 & .060 & -11.355100 & .115100 \\
\hline
\end{tabular}

Table 3: Bonferroni post hoc test to evaluate the groups showing a significant difference from the golden proportion. 


\section{Discussion}

The study was performed to evaluate the proportion of the maxillary anterior teeth in patients with narrow, average and wide smiles and their variation from the golden proportion.

The sample consisted of thirty north Indian patients with esthetically pleasing smiles.

The proportions of the widths of maxillary anterior teeth were calculated. Only the proportions of the photographs affected the results and not the size. The proportions of the photographs were kept constant i.e. $4 \times 6$.

According to Preston [6], golden proportion is in the range of $0.61-0.63$, and the same range was considered in this study during evaluation of the data.

All the three groups showed a highly significant difference from the golden proportion.

The prevalence of golden proportion in the past has been determined for different ethnic groups in different studies.

Some researchers have advocated the use of this mathematical proportion while some have opted against its use. Preston found $17 \%$ of his study samples had golden proportion between the width of the maxillary central and lateral incisors. Lombardi recommended a repeated ratio concept in contrast to golden proportion. Mahshid., et al. reported that the golden proportion did not exist between the widths of the maxillary anterior teeth and it was supported by Ward, Gillen., et al. Rosenstiel., et al. [15] Aziz [9] studied the Bangladeshi population and concluded that the golden ratio may not be present in all ethnicities and adjusted golden percentage might serve as a guideline to create harmonious proportions. According to Chander., et al. [15] the golden proportion is an inappropriate method to relate the successive widths of the maxillary anterior teeth in Indian population.

According to Hegde [10] Golden proportion was not prevalent in the south Indian population and did not serve as an adequate guideline to be applied to all maxillary anterior teeth.

All these conclusions by different authors are in agreement with this study.

The distance between the lateral junction of the upper and lower lips and the distal points of the canines during smiling is known as the "buccal corridor." As light passes posteriorly, it is reduced and thus gives the teeth a darker shade and therefore a smaller appearance [11]. Hulsey compared the attractiveness of orthodontically treated and untreated smiles. He examined the "buccal corridors" as a ratio: distance between the maxillary canines/distance between the corners of the smile. He then observed that the buccal corridor ratio was of no significance to an attractive smile [16]. According to Tikku., et al. increased buccal corridor display was considered less attractive by laypersons and orthodontists alike [17].

This study did not assess the smile score but evaluated the smile width by the criteria given by Profit. According to Profit, the buccal corridor width in ideal smile was $13-17 \%$ of the inter-commissure distance, less than $13 \%$ in wide smiles and greater than $17 \%$ in narrow smiles [14].

The data suggested that the greatest variation of the mean values from the golden proportion was found in the wide smile group followed by the narrow smile group and average smile group.

The canine proportion values deviated greater from the golden proportion as compared to the lateral incisor proportion values. The deviation of canine proportion also followed the same sequence, i.e wide smile group followed by the narrow smile group and average smile group.

Calculating the proportions involved measuring the apparent width of the teeth.

According to Hyung., et al. the buccal corridor is a multifactorial phenomenon, affected by lateral cephalometric as well as cast variables. FMA, lower anterior facial height, upper incisor (U1) exposure, U1 to facial plane, lower incisor (L1) to mandibular plane, L1 to N-B, Sn (subscale) to soft tissue mention ( $\mathrm{Me}^{\prime}$ ), Sn to stomodeum superius (stms), stems to $\mathrm{Me}^{\prime}$, and interpersonal width were significantly correlated with Buccal corridor area [13]. The proportion of anterior teeth is calculated by measuring the apparent width of teeth as viewed on the photograph. The teeth form an arch form. The visibility of the anterior teeth is affected by the arc formed by the anterior teeth. One can expect the apparent width of the lateral and canine to be greater in wide arch and less in narrow arch form. The width of central incisors remains constant as it is the dominant tooth in the smile arc and fully visible. The results of the study do support the view to some extent. The greatest deviation was observed in the wide smile, where a wide arch form can be expected. This was followed by a narrow smile, where the discrepancy could be attributed to comparative narrow width of the central incisors or the narrow apparent width of the lateral incisor as compared to canine. The closest values were observed in the patients with average smile. In general, this study and several other surveys have estimated that there is no golden standard in the nature. It may be required to define a new proportion according to different ethnicities to define an esthetic smile and aid in smile designing during treatment. If the value closest to golden proportion is considered to be esthetic, the average smile would be the goal of the treatment. Many surveys have shown that wide smile is considered to be the most esthetic, therefore further studies have to be done to correlate the variables. The gender differences in the 
variation of golden proportion were not investigated in the study. Further studies can investigated these differences and aid in smile designing and improve the treatment planning.

\section{Conclusion}

From the present study, the following conclusions were drawn:

The maxillary anterior teeth the population in question did not follow the golden proportions.

The wide smile group showed the greatest variation from the golden proportion followed by narrow smile group and average smile group.

The canine showed a greater variation as compared to lateral incisor irrespective of the smile width.

\section{Conflict of Interest}

There is no conflict of interest.

\section{Bibliography}

1. Vegter F and Hage JJ. "Clinical anthropometry and canons of the face in historical perspective". Plastic and Reconstructive Surgery 106 (2000): 1090-1096.

2. Machado AW. "10 commandments of smile esthetics, Dental press". Journal of Orthodontics 19.4 (2014): 136-157.

3. Golden proportion: A review S.M. Laxmikanth, S.R. Raghavendra Journal of Advanced Clinical and Research Insights 1.1 (2014).

4. Sreenivasan Murthy BV and Ramani N. "Evaluation of natural smile: Golden proportion, RED or Golden percentage". Journal of Conservative Dentistry 11.1 (2008): 16-21.

5. Morley J. "Smile design specific considerations". Journal of the California Dental Association 25.9 (1997): 633-637.

6. Preston JD. "The golden proportion revisited". Journal of Esthetic Dentistry 5.6 (1993):247-251.

7. Levin EL. "Dental esthetics and the golden proportion". Journal of Prosthetic Dentistry 40 (1978): 244-252.

8. Gillen RJ., et al. "An analysis of selected normative tooth proportion". The International Journal of Prosthodontics 7.5 (1994): 410-417.

9. Aziz M and Hossain MZ. "Validity of mathematical proportions in maxillary anterior teeth among Bangladeshi population". APOS Trends in Orthodontics 7 (2017): 41-48.

10. Hegde MN and Malhotra S. "Evaluation of golden proportion between maxillary anterior teeth of south indian population". The Open Dentistry Journal 2.5 (2016): 137- 141.
11. Lombardi RE. "The principles of visual perception and their clinical application to denture esthetics". Journal of Prosthetic Dentistry 29.11 (1973): 358-382.

12. Roden-Johnson D., et al. "The effects of buccal corridor spaces and arch form on smile esthetics". American Journal of Orthodontics and Dentofacial Orthopedics 127.3 (2005): 343-350.

13. Il-Hyung Yang., et al. "Which Hard and Soft Tissue Factors Relate with the Amount of Buccal Corridor Space during Smiling?". The Angle Orthodontist (2008): 5-11.

14. Profitt W R. “Contemporary Orthodontics, 5thed. St Louis' Mosby" (2013).

15. Chander NG., et al. "Golden proportion assessment between maxillary and mandibular teeth on Indian population". The Journal of Advanced Prosthodontics 4.2 (2012): 72-75.

16. Hulsey CM. "An esthetic evaluation of lip-teeth relationships present in the smile". American Journal of Orthodontics 57 (1970): 132-144.

17. Tikku T., et al. "Role of buccal corridor in smile esthetics and its correlation with underlying skeletal and dental structures". Indian Journal of Dental Research 23.2 (2012): 187-194.

Volume 3 Issue 12 December 2019

(C) All rights are reserved by Shubhangi Jain., et al. 